\title{
Ueber den Zusammenhang zwischen Gedanken, Erkenntniswert und Oratio obliqua bei Frege*
}

\author{
Kazuyuki Nомото
}

In diesem Aufsatz möchten wir den Zusammenhang zwischen Sinn, Erkenntniswert und Oratio Obliqua bei Frege klar machen.

Zunächst berühren wir Kriterium Freges für die Gedankengleichheit. Obwohl Frege allerdings nicht so explizit auf das positive Kriterium für die Gedankengleichheit, sondern nur auf gewisse negative oder notwendige Bedingungen für sie hinzuweisen scheint, kann man mindestens zwei verschiedene Kriterien für die Gedankengleichheit in den Werken Freges unterscheiden.

\section{Das intensionale Kriterium}

\subsection{Die logische Aequivalenz}

Frege hat bereits in seiner Begriffsschrift die folgende Bedingung für die Gleichheit des beurteilbaren Inhalts bzw. des begriffichen Inhalts eines Urteils angeführt:

... bemerke ich, daß die Inhalte von zwei Urteilen in doppelter Weise verschieden sein können: erstens so, daß die Folgerungen, die aus dem einen in Verbindung mit besimmten andern gezogen werden können, immer auch aus dem zweiten in Verbindung mit denselben andern Urteilen folgen; zweitens so, daß dies nicht der Fall ist... Ich nenne nun denjenigen Teil des Inhaltes, der in beiden derselbe ist, den begrifflichen Inhalt ... weil im Urteile hier nur das in Betracht kommt, was auf die möglichen Folgerungen Einfluss hat.

(BS. 2-3)

Wenn man also den Gedanken mit dem begrifflichen Inhalt identifizieren kann, kann man diese Gleichheit der „Folgerungsmengen“ als ein Kriterium für die Gedankengleichheit ansehen.(1)

Wie Thiel bemerkt, ${ }^{(2)}$ sieht Frege außerdem die logische Aequivalenz klar als ein Kriterium für die Gedankengleichheit an, und zwar in dem Briefe an Husserl an. ${ }^{(3)}$ Man kann also folgender Äußerung Bartletts zustimmen : (4)

Zwei Sätze drücken denselben Gedanken aus, genau dann wenn sie zwar logisch äquivalent sind, aber weder sie noch irgendwelche Teile von ihnen logisch evidenz (determinent) sind.

Hokkaido University, Faculty of Letters, Department of Philosophy, Kita 10, Nishi 7, Kita-ku, Sapporo 060, Japan 
Wir möchten nun die logische Aequivalenz normaller Weise wie folgt definieren :

Beide Sätze,$\phi^{\prime}$ und,$\psi^{\prime}$ sind nur logisch äquivalent genau dann wenn $\ulcorner\phi$ $\leftrightarrow \psi\urcorner$ logisch allgemeingültig ist, d.h. wenn die Wahrheit des Satzes $\ulcorner\phi \leftrightarrow$ $\psi\urcorner$ nur auf der Basis der semantischen Regeln des semantischen Systems, ohne Bezugnahme auf (außerlinguistische) Fakten, beruht bzw. wenn $\ulcorner\phi \leftrightarrow$ $\psi\urcorner$ wahr in jedem semantischen Modell ist ${ }^{(5)}$,

Carnap führt dann die folgende Konvention ein :

Die zwei Designatoren haben dieselbe Intension genau dann wenn sie einander logisch äquivalent sind. Die Intension eines Satzes wird Proposition genannt und die Intension eines singulären Terminis wird individueller Begriff (individual concept) genannt ${ }^{(6)}$

\subsection{Die notwendige Wahrheit}

In der in jungster Zeit entwickelten modalen Logik werden die beiden Begriffe der logischen Allgemeingültigkeit und der notwendigen Wahrheit scharf voneinander unterschieden. ${ }^{(7)}$ Man kann aber sagen, daß $\ulcorner\phi \leftrightarrow \psi\urcorner$ notwendig wahr ist, wenn $\ulcorner\phi \leftrightarrow \psi\urcorner$ logisch allgemeingültig ist, denn die Regel der modalen Verallgemeinerung ist allgemeingültig. Man kann deshalb eine andere Form der notwendigen Bedingung für die Gedankengleichheit als Proposition erhalten. Das heißt:

Wenn $\ulcorner\square(\phi \leftrightarrow \psi)\urcorner$ nicht wahr ist, dann ist $\ulcorner\phi \leftrightarrow \psi\urcorner$ nicht logisch all-

gemeingültig, d.h.,$\phi^{\prime}$ und,$\psi^{\prime}$ sind nicht logisch äquivalent und drücken also verschiedene Propositionen aus.

Man könnte diese notwendige Bedingung für die Gleichheit der Proposition ein modales Kriterium nennen.

\section{Das epistemische Kriterium}

\subsection{Die Verschiendenheit im Gedanken}

Frege betrachtet die logische Aequivalenz nicht als ein befriedigendes Kriterium für die Gedankengleichheit. ${ }^{(8)}$ Wenn beide Sätze auch logisch äquivalent sein mögen, könnten die Gedanken dieser beiden Sätze doch verschieden sein. Die logische Aequivalenz ist also nur eine notwendige, aber nicht hinreichende Bedingung für die Gedankengleichhet im Sinne Freges.

Frege bietet infolgedessen eine neue engere notwendige Bedingung für Gedankengleichheit an, d.h. wenn man ein Wort in einem Satz durch ein anderes von derselben Bedeutung, aber verschiedenem Sinne ersetzt, dann ändert sich der Gedanke, d.h. jeder entsprechende Bestandteil in den beiden Sätzen muss denselben Sinn haben, damit sie denselben Gedanken ausdrücken, d.h. 


$$
\bar{\Delta} \neq \bar{A} \overline{\phi(\Delta)} \neq \overline{\phi(A)}{ }^{(9)}
$$

(Im allgemeinen drückt $\bar{\alpha}$ den Sinn bzw. die Proposition des Ausdrucks $\alpha$ aus.)

\subsection{Die intensionale Isomorphie}

Wenn man Freges Unterscheidung der gewöhnlichen und ungeraden Bedeutung und die Einführung der Wendung ,der Sinn des Ausdrucks ,a““ zulässt, dann kann man das sogenannte Prinzip der Ersetzbarkeit von der Bedeutung auf den Sinn übertragen, und man bekommt eine neue hinreichende Bedingung für Gedankengleichheit wie folgt:

Wenn man ein Zeichen , $A^{6}$ in , $\phi(A)^{\prime}$ durch ein anderes , $A^{\prime}$ von demselben Sinn wie , $A^{\text {‘ }}$ ersetzt, so drückt , $\phi(\Delta)^{\text {‘ }}$ denselben Gedanken wie,$\phi(A)^{`}$ aus, d.h. $A=\bar{\Delta} \rightarrow \overline{\phi(A)}=\overline{\phi(\Delta)}{ }^{(10)}$

Wenn man diese Bestimmung akzeptiert, dann darf man sagen, daß die beide Sätze denselben Gedanken ausdrücken genau dann wenn jeder entsprechende Bestandteil der Sätze denselben Sinn hat, d.h.

$$
\bar{\Delta}=\bar{A} \leftrightarrow \overline{\phi(\Delta)}=\overline{\phi(A)} .
$$

Carnap hat diese engere Bedingung für die Gedankengleichheit intensionale Isomorphie genannt und sie wie folgt definiert: (11)

Zwei einfache Designatoren sind intensional isomorph bzw. haben dieselbe intensional Struktur genau dann wenn sie logisch äquivalent sind. Zwei komplexe Designatoren sind genau dann intensional isomorph bzw. haben dieselbe intensionale Struktur, wenn jeder Bestandteil des Designators intensional isomorph mit dem entsprechenden Bestandteil des anderen Designators und wenn die Anordnng der entsprechenden Bestandteile in beiden Designatoren dieselbe ist.

Man kann also hier zunächst mindestens zwei Kriterien für die Gleichheit vom Fregeschen Gedanken unterscheiden :

(A) Beide Sätze , $\phi^{\prime}$ und , $\phi^{6}$ drücken dieselbe Proposition aus, wenn , $\phi^{\text {‘ }}$ und,$\psi^{6}$ logisch äquivalent sind bzw. wenn $\ulcorner\phi \leftrightarrow \psi\urcorner$ notwendig wahr ist.

Dieses Kriterium kann intensional genannt werden.

(B) Beide Sätze,$\phi^{6}$ und,$\psi^{6}$ haben dieselbe intensionale Struktur oder drücken denselben Gedanken im engeren Sinne aus genau dann wenn , $\phi^{\text {c }}$ und,$\psi^{6}$ intensional isomorph im Sinne Carnaps sind.

Dieses Kriterium kann epistemisch genannt werden, weil die intensionale Isomorphie im Fall Freges mit solchen epistemischen Elementen wie Erkenntniswert, Erkenntnistat, der Art des Gegebenseins und Oratio obliqua in engen Zusammenhang steht. 


\section{Der Sinn, die Art des Gegebenseins des Bezeichneten, der Erkenntniswert, die Erkenntnistat und die Oratio obliqua}

Wir möchten nun den Zusammenhang zwischen dem Sinn, der Art des Gegebenseins, dem Erkenntniswert und der Erkenntnistat klar machen. Nehmen wir an, daß die folgenden beiden Identitätssätze wahr sind :

(i) $a=a$,

(ii) $a=b$.

\subsection{Der Sinn und die Art des Gegebenseins}

1) Sinn

Nehmen wir an, daß der Sinn von, $a^{6}$ von dem von , $b^{6}$ verschieden ist. Die Sätze (i) und (ii) sind dann auch voneinander im Gedanken verschieden. Wir drücken den Sinn von (i), (ii) und von, $a^{6}, b^{6}$ als $\overline{(\mathrm{i})}, \overline{(\mathrm{ii})}$ und $\bar{a}, \bar{b}$ aus. Dann (i) $\neq \overline{(i i)}$, weil $\bar{a} \neq \bar{b}$.

2) Die Art des Gegebenseins

Nach Frege entspricht,,der Unterschied des Zeichens einem Unterschiede in der Art des Gegebenseins des Bezeichneten.“ (SuB. 26) „Es muß also eigentlich gefordert werden, daß mit jedem Eigennamen eine einzige Weise verknüpft sei, wie der, die oder das durch ihn Bezeichnete gegeben sei. " (Ged. 66) Wir drücken die Art des Gegebenseins des Bezeichneten von (i), (ii) und von, $a^{6}, b^{6}$ als $A(\mathrm{i}), A(\mathrm{ii})$ und $A(a)$, $A(b)$ aus. Dann $A(\mathrm{i}) \neq A(\mathrm{ii})$, weil $A(a) \neq A(b)$.

Der Sinn eines Zeichens oder der Gedanke eines Satzes entspricht sehr eng der Art des Gegebenseins des Bezeichneten. In der Tat hält Frege den Sinn des Zeichens für das, ,worin die Art des Gegebenseins enthalten ist." (SuB. 26) So ist es auch im Fall des Gedankens eines Satzes. ${ }^{(12)}$

Man kann also sagen, daß die epistemische Bestimmungsweise eines Objekts sprachlich im Sinn eines Zeichens eingebettet wird, das das Objekt bezeichnet, d.h. die Art und Weise des Gegebenseins des Objekts ist im Sinn des Zeichens enthalten, das das Objekt bezeichnet. Man kann deshalb sagen, daß (i) und (ii) im Sinn voneinander verschieden sind genau dann wenn (i) und (ii) voneinander in der Art und Weise des Gegebenseins des Bezeichneten (d.h. des Wahrheitswerts) differieren, d.h.,

Prinzip (I) : $\overline{(\mathrm{l})} \neq(\overline{\mathrm{ii}})$ genau dann wenn $A(\mathrm{i}) \neq A(\mathrm{ii})$.

\subsection{Der Erkenntniswert und die Erkenntnistat}

3) Erkenntnistat

Frege sagt:

Die Wörter „Morgenstern“ und „Abendstern“ bezeichnen denselben 
Planeten Venus ; aber um dies zu erkennen, ist eine besondere Erkenntnistat erforderlich. (Brief an Russell, am 28.12.1902)

Man könnte also sagen, daß es einer besonderen Erkenntnistat bedarf, um die Wahrheit des Satzes (ii) einzusehen (man kann dies als $\mathrm{T}^{+}$(ii) ausdrücken), andererseits scheint keine besondere Erkenntnistat erforderlich zu sein, um die Wahrheit von (i) zu erkennen (man kann dies als $\mathrm{T}^{-}$(i) ausdrücken.).

4) Erkenntniswert

Wir drücken den Erkenntniswert von (i) und (ii) als $E$ (i) und $E$ (ii) aus, dann kann man wie folgt formulieren: $E($ i $) \neq E$ (ii), weil (i) und (ii) nach Frege verschieden im Erkenntniswert sind.

Bei der Erklärung der Verschiedenheit im Erkenntniswert von , $a=a^{“}$ und

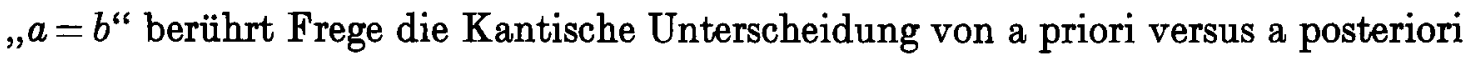

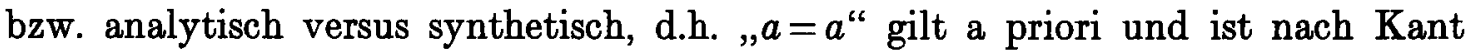

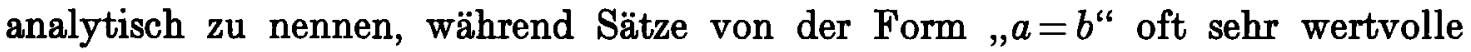
Erweiterungen unserer Erkenntnis enthalten und nicht immer a priori zu begründen sind. (SuB. 25)

Kann man nun im allgemeinen sagen, daß die Verschiedenheit im Erkennt niswert von zwei Sätzen dem Kantischen Unterschied entspricht, derart, daß der eine analytisch (d.h. nur erläuternd) a priori gilt, während der andere synthetisch (d.h. erweiternd) und nicht immer a priori zu begründen ist ?(13)

Frege gibt allerdings eigene Bestimmungen von solchen Unterscheidungen, die man mit dem Kantischen Kriterium nicht völlig identifizieren kann. ${ }^{(14)}$ Freges eigener Unterschied zwischen den analytischen und synthetischen Sätzen beruht darauf, ob sie nur aus den allgemeinen logischen Gesetzen und Definitionen folgen oder nicht. Andererseits beruht Freges Unterscheidung zwischen den a priori- und a posteriori-Sätzen auf der Frage, ob ihre Beweise nur auf Grund allgemeiner Gesteze geführt werden oder von Tatsachen abhängen. Man kann also nach Frege die beweisbare Sätze wie folgt klassifizieren :

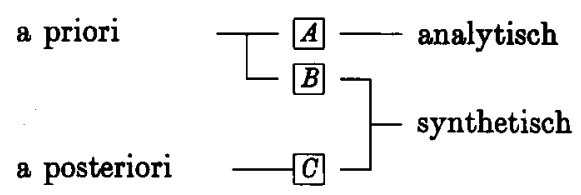

Die Gruppen $A$ und $B$ der a priori-Sätze bestehen aus den Sätzen, die aus den unbewisbaren unmittelbar einleuchtenden allgemeinen Gesetzen bewiesen werden können. Die Gruppe $C$ der a posteriori-Sätze besteht aus den Sätzen, die aus den unbeweisbaren aber nicht allgemeinen, unmittelbar klaren tatsächlichen Wahrheiten bewiesen werden können. Die Gruppe $A$ der analytische Sätze besteht aus den Sätzen, die aus den allgemeinen logischen Gesetzen und Definitionen bewiesen werden können. Die Gruppe $B$ und $C$ der synthetischen Sätze bestehen aus sol- 
chen, die eines besonderen Wissensgebiets bedürfen. Die Sätze in $B$ leiten sich aus den allgemeinen Gesetzen im besonderen Wissensgebiet, während die Sätze in $C$ ganz auf den besonderen tatsächlichen Wahrheiten basieren. Zum Beispiel gehören die Sätze aus der Logik und Arithmetik, nach Frege, zur Gruppe $A$, d.h. sie sind analytisch a priori. Die Sätze aus den Geometrie und reinen Mechanik usw. gehören zur Gruppe $B$, d.h. sie sind synthetisch a priori. Man muss bemerken, daß diese Klassifikation das Fundament des Beweises eines beweisbaren Satzes betrifft. Es handelt sich hierbei um beweisbare Sätze auf unbeweisbaren, unmittelbar einleuchtenden Urwahrheiten.

Die Frage, ob ein Satz eine wertvolle Erweiterung unserer Erkenntnis enthält, d.h. ob er synthetisch im Sinne Kants ist, scheint allerdings unabhängig davon zu sein, ob der Satz im Sinne Freges a priori bzw. a posteriori und analytisch bzw. synthetisch ist.

Frege gibt ein eindrucksvolles Bespiel aus der Arithmetik. Der arithmetische Satz , $3+1=2.2^{\prime \prime}$ ist zwar nach Frege ebenso analytisch a priori wahr wie ,2.2 $=2.2^{\prime \prime}$, aber der Sinn von $3+1^{6}$ ist verschieden von dem Sinn von ,2.2'. (Peano, 369) Der Satz enthält also eine bedeutsame Erweiterung unserer Erkenntnis. In der Tat ist eine besondere Erkenntnistat erforderlich, um seine Wahrheit einzusehen, während ,2.2=2.2" nichts anderes als die selbstverständliche Anwendung des Identitätsprinzips ist. Die beiden Sätze sind also im Erkenntniswert voneinander verschieden, obwohl sie nach Frege analytisch a priori sind.

Man kann also mindestens sagen, daß die Verschiedenheit im Erkenntniswert nicht im unmittelbaren Zusammenhang mit Freges Unterscheidung hinsichtlich der Begründung, also mit den Begriffspaaren ,analytisch“-,,synthetisch“ bzw. ,a priori“-,a posteriori“" steht. Freges Unterscheidung von analytisch-synthetisch bzw. a priori-a posteriori betrifft das Fundament des Beweises eines Satzes, während die Verschiedenheit im Erkenntniswert vielmehr davon abhängig ist, ob der Satz eine bedeutsame Erweiterung unserer Erkenntnis enthält, d.h. ob eine besondere Erkenntnistat erforderlich ist, um seine Wahrheit einzusehen. Es gibt also einen sehr engen Zusammenhang zwischen der Verschiedenheit im Erkenntniswert und der Forderung nach Notwendigkeit einer besonderen Erkenntnistat. Das heißt, Freges Unterscheidung im Erkenntniswert von Sätzen scheint sich nicht auf seine eigene präzise Unterscheidung hinsichtlich der möglichen Begründung von Sätzen, sondern vielmehr auf die Kantische Unterscheidung von analytisch-synthetisch zu beziehen. Wenn ein Satz nur erläuternd (analytisch a priori im Kantischen Sinne) wahr ist, während ein anderer erweiternd (synthetisch a posteriori oder a priori) wahr ist, könnte man sofern sagen, daß die beide Sätze voneinander im Erkenntniswert verschieden sind. Wenn es keiner besonderen Erkenntnistat bedarf, um die Wahrheit eines Identitätssatzes zu erkennen, während eine besondere Erkenntnistat erforderlich ist, um die Wahrheit eines anderen Identitätssatzes einzusehen, könnte 
man also sagen daß die beide Sätze im Erkenntniswert voneinander verschieden seien, d.h.,

Prinzip (II) : $E(\mathrm{i}) \neq E\left(\right.$ ii), wenn $T^{+}(\mathrm{ii})$, während $T^{-}(\mathrm{i})$.

Wir können nun bestätigen, daß ein enger Zusammenhang zwischen dem Sinn und der Art des Gegebenseins existiert (d.h. Prinzip (I)) und andererseits auch zwischen dem Erkenntniswert und der Erkenntnistat (d.h. Prinzip (II)).

\subsection{Sinn, Erkenntniswert und Erkenntnistat}

Frege behauptet außerdem, daß der Sinn eines Satzes mit dem Erkenntniswert des Satzes und mit der Notwendigkeit einer besonderen Erkenntnistat, um seine Wahrheit zu erkennen, in engem Zusammenhang steht. Frege sagt wie folgt:

Ueberall, wo das Zusammenfallen der Bedeutung nicht selbstverständlich ist, haben wir eine Verschiedenheit des Sinnes. So ist auch der Sinn von $\gg 2^{3}+1 \ll$ verschieden von dem Sinne von $\gg 3^{2} \ll$, obwohl wir dieselbe Bedeutung haben, weil eine besondere Tat des Erkennens erforderlich ist, um dies einzusehen. So sind auch die Gleichungen $\gg 3^{2}=3^{2} \ll$ und $\gg 2^{3}+$ $l=3^{2} \ll$ für die Erkenntnis nicht gleichwertig, obwohl der Wahrheitswert derselbe ist. Der Unterschied ist der des Sinnes; die ausgedrückten Gedanken sind verschieden... (Brief an Russell, am 28.12.1902)

Ich sage in solchem Falle: beide Bezeichnungen haben zwar dieselbe Bedeutung, bezeichnen oder bedeuten oder benennen dasselbe, aber sie haben verschiedenen Sinn, weil es einer besonderen Erkenntnistat bedarf, um das Zusammenfallen einzusehen. (Peano, 369)

Hier wird der Grund bzw. die Ursache der Verschiedenheit des Sinnes in der Tatsache gesehen, daß eine besondere Tat des Erkennens erforderlich ist, um die

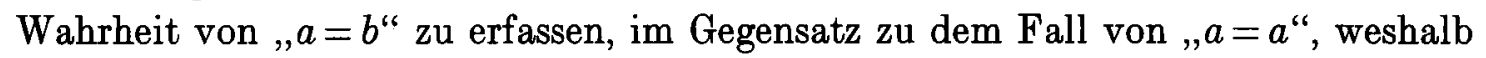
die beide Sätze nach dem Prinzip (II) verschieden im Erkenntniswert sind. Man könnte also sagen, daß die Verschiedenheit im Erkenntniswert sozusagen die ratio cognoscendi der Verschiedenheit des Sinnes ist. Mit anderen Worten, wenn eine besondere Erkenntnistat erforderlich ist, um die Warheit eines Identitätssatzes (ii) einzusehen, während es keiner solchen bedarf, um die Wahrheit eines anderen Satzes (i) zu erkennen, und also (i) und (ii) im Erkenntniswert voneinander verschieden sind, dann kann man sagen, daß (i) und (ii) im Hinblick auf den Sinn voneinander verschieden sind. Die Notwendigkeit oder Nichtnotwendigkeit einer besonderen Erkenntnistat, zu dem Zweck, die Wahrheit von Sätzen einzusehen und also die Verschiedenheit im Erkenntniswert von Sätzen, ist wahrscheinlich die hinreichende Bedingung als die ratio cognoscendi für die Verschiedenheit des Sinnes von Sätzen, 
d.h.,

Prinzip (III) $)_{1}$ : wenn $E(\mathrm{i}) \neq E(\mathrm{ii}), \operatorname{dann} \overline{(\mathrm{i})} \neq \overline{(\mathrm{ii})}$.

Außerdem erklärt Frege umgekehrt die Verschiedenheit im Erkenntniswert durch die Verschiedenheit des Sinnes:

Eine Verschiedenheit [im Erkenntniswert von , $a=a^{\prime \prime}$ und , $\left.a=b^{\prime \prime}\right]$ kann nur dadurch zustande kommen, daß der Unterschied des Zeichens einem Unterschiede in der Art des Gegebenseins des Bezeichneten [und also einem Unterschied des Sinnes] entspricht.

(SuB. 26)

Wenn wir den Erkenntniswert von , $a=a^{\prime \prime}$ und , $a=b^{\prime \prime}$ im allgemeinen verschieden fanden, so erklärt sich das dadurch, daß für den Erkenntniswert der Sinn des Satzes, nämlich der in ihm ausgedrückte Gedanke, nicht minder in Betracht kommt als seine Bedeutung, das ist sein Wahrheitswert. Wenn nun $a=b$ ist, so ist zwar die Bedeutung von „, $b$ " dieselbe wie die von „, $a$ " und also auch der Warhheitswert von , $a=b$ " derselbe wie von , $a=a^{“}$. Trotzdem kann der Sinn von , $b$ “ von dem Sinn von , $a$ “ verschieden sein, und mithin auch der in , $a=b^{\prime \prime}$ ausgedrückte Gedanke verschieden von dem [in] , $a=a^{\prime \prime}$ ausgedrückten sein; dann haben beide

Sätze auch nicht denselben Erkenntniswert.

(SuB. 50)

Die mögliche Verschiedenheit im Erkenntniswert von „ $a=a^{\prime \prime}$ und „, $a=b^{\prime \prime}$ wird hierbei in der Tat durch die mögliche Verschiedenheit des Sinnes von , $a$ " und , $b$ “ erklärt. Die Verschiedenheit des Sinnes von beiden Sätzen ist sozusagen die ratio essendi der Verschiedenheit im Erkenntniswert von Sätzen. Wenn der in , $a=b^{\text {“ }}$ ausgedrückte Gedanke verschieden von $\operatorname{dem}$ in , $a=a^{\text {" }}$ ausgedrückten ist, dann haben beide Sätze auch nicht denselben Erkenntniswert. Man könnte annehmen, Freges eigentümliche Auffassung liege darin, da $\beta$ er die epistemologische Verschiedenheit im Erkenntniswert durch die sprachlich-semantische Verschiedenheit des Sinnes zu erklären versucht. Die epistemologische Verschiedenheit im Erkenntniswert von Sätzen, d.h. die Notwendigkeit bzw. Nichtnotwendgkeit einer besonderen Erkenntnistat, um ihre Wahrheit zu erkennen und außerdem die verschiedene Art und Weise des Gegebenseins des Bezeichneten würden sich nach einer solchen Auffassung sozusagen in der sprachlich-semantischen Verschiedenheit ihres Sinnes spiegeln. Es scheint also das folgende Prinzip allgemein zu gelten:

Prinzip (III) $)_{2}$ : wenn $\overline{(\mathrm{i})} \neq \overline{(\mathrm{ii})}$, dann $E(\mathrm{i}) \neq E(\mathrm{ii})$.

Aber man kann dafür nicht wirklich allgemeine Geltung beanspruchen, daß die Verschiedenheit des Sinnes die hinreichende Bedingung als die ratio essendi für die 
Verschiedenheit im Erkenntniswert ist.

Wir möchten zuerst einige Gegenbeispiele zu dem genannten Prinzip (III) vorstellen. Die beide Sätze z.B. „Der Morgenstern ist der Morgenstern“ und „Der Abendstern ist der Abendstern" dürfen nicht im Erkenntniswert voneinander verschieden sein, weil sie nach Frege und Kant analytisch a priori sind. Andererseits ist es aber klar, daß der Gedanke des einen Satzes ganz differiert von dem des anderen ist, denn der Sinn von ,der Morgenstern“ ist verschieden von dem von „der Abendstern". Ebenso verhält es sich bei den Sätzen , $1+3=1+3$ " und, $2^{2}=2^{2 \text { " }}$.

Außerdem drücken zwei Sätze wie z.B. ", Der Morgenstern ist der Abendstern“ und „Der Morgenstern ist der Planet Venus" unterschiedliche Gedanken aus, obwohl sie synthetisch a posteriori wahr sind und also denselben Erkenntniswert haben. Solange man das Prinzip (II) als das Kriterium für die Gleichheit des Erkenntniswerts ansieht, kann man den Erkenntniswert des Satzes „Der Morgenstern ist der Morgenstern“ nicht von dem Satzes „Der Abendstern ist der Abendstern“ unterscheiden. Bloße Verschiedenheit des Sinnes kann also nicht im allgemeinen die hinreichende Bedingung (als die ratio essendi) für die Verschiedenheit des Erkenntniswerts sein, d.h. das Prinzip (III) $)_{2}$ wird nicht verallgemeinern.

Kann man aber sagen, daß die Verschiedenheit des Erkenntniswerts uneingeschränkt die hinreichende Bedingung als die ratio cognoscendi für die Verschiedenheit des Sinnes ist? Das heißt, kann man das Prinzip (III) $)_{1}$ wirklich fraglos behaupten? Man könnte die Gegenbeispiele dafür im Fall jener Sätze finden, in denen ,token-reflexive'-Ausdrücke wie ,ich“, ,jetzt", „hier", „heute“, „gestern“, "dies" usw. enthalten sind.(15) Man müßte sagen, daß das Prinzip (III) ${ }_{1}$ allgemeingültig ist, sofern beide Sätze (i) und (ii) keine ,token-reflexive'-Ausdrücke enthalten.

\subsection{Gedanke und Oratio obliqua}

Es gibt außerdem einen Zusammenhang zwischen dem Erkenntniswert, der Oratio obliqua und dem Gedanken. Ich möchte z.B. zwei Passagen zitieren, in denen Frege den Zusammenhang zwischen dem Gedanken, der Ortatio obliqua und also implizit dem Erkenntniswert erörtert. Die erste lautet wie folgt :

$\left(^{*}\right)$ Ersetzen wir nun in ihm [dem Satz] ein Wort durch ein anderes von derselben Bedeutung, aber anderem Sinne, so kann dies auf die Bedeutung des Satzes keinen Einfluß haben. Nun sehen wir aber, daß der Gedanke sich in solchem Falle ändert; denn es ist z.B. der Gedanke des Satzes ,,der Morgenstern ist ein von der Sonne beleuchteter Körper" verschieden von dem Satz „der Abendstern ist ein von der Sonne beleuchteter Körper". Jemand, der nicht wüßte, daß der Abendstern der Morgenstern ist, könnte den einen Gedanken für wahr, den anderen für falsch halten. (SuB. 32) Die zweite lautet folgendermaßen :

$\left({ }^{* *}\right)$ Nun ist es möglich, daß Herbert Garner den Sinn des Satzes „Dr. 
Lauben ist verwundet worden“ für wahr hält, während er, durch falsche Nachrichten irregeführt, den Sinn des Satzes „Gustav Lauben ist verwundet worden" für falsch hält. Unter den gemachten Annahmen sind diese Gedanken also verschieden. (Ged.65)

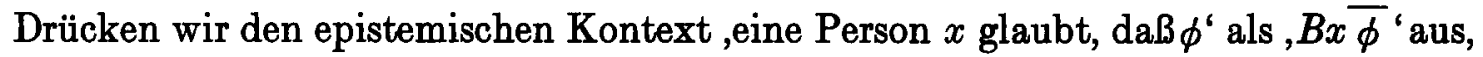
dann könnten die oben genannten Behauptungen Freges zusammen wie folgt formuliert werden :

Prinzip (IV) : $(\bar{\phi} \neq \bar{\phi}) \longleftrightarrow \diamond \exists x(B x \bar{\phi} \&\urcorner B x \bar{\phi})$

Dieses Prinzip scheint allgemeingültig sein, sofern die Sätze , $\phi^{\prime}$ und , $\psi^{\prime}$ keine ,token reflexive-Ausdrücke enthalten. Aber der folgende Teil des Prinzips (IV), der dem $\left({ }^{* *}\right)$ entspricht, scheint zu stark und also nicht allgemeingültig zu sein, wenn die Sätze,$\phi^{6}$ und,$\phi^{6}$,token reflexive'-Ausdrücke enthalten, weil es dafür ein Gegenbeispiel gibt, z.B. drücken (1) und (2) nach Frege denselben Gedanken aus, aber es ist möglich, daß jemand (1) für wahr hält, während er (2) für falsch hält.. ${ }^{(16)}$

$$
\begin{aligned}
(\mathrm{IV})^{* *}: & \diamond \exists x(B x \bar{\phi} \&\urcorner B x \bar{\psi}) \longrightarrow(\bar{\phi} \neq \bar{\psi}) \\
& \quad \text { oder }(B a \bar{\phi} \&\urcorner B a \bar{\phi}) \longrightarrow(\bar{\phi} \neq \bar{\psi})
\end{aligned}
$$

(1) Heute [am 10.8.1980 ausgesprochen] war es warm in Göttingen.

(2) Gestern [am 11.8.1980 ausgesprochen] war es warm in Göttingen.

Was das Prinzip (IV) angeht, wird also die Möglichkeit der Verschiedenheit im Glaubenshaltung, die vielleicht aus der Verschiedenheit im Erkenntniswert von Sätzen herrührt, nicht als die hinreichende, sondern nur als eine notwendige Bedingung (als die ratio cognoscendi) für die Verschiedenheit des Sinnes betrachtet, wenn die beide Sätze,$\phi^{\prime}$ und,$\phi^{6}$, token reflexive' Ausdrücke enthalten. Das heißt, die Verschiedenheit des Sinnes ist nicht das einzige semantische Gegenstück für die Möglichkeit der Verschiedenheit in der Glaubenshaltung und vielleicht im Erkenntniswert, wenn , $\phi^{6}$ und,$\psi^{6}$, token reflexive'-Ausdrücke enthalten. Also wenn indexikalische Ausdrücke und Demonstrativa in Sätzen enthalten sind, würde es einer stärkeren Bedingung für Synonymie bedürfen. ${ }^{(17)}$

Man könnte die Zusammenhänge zwischen Sinn, Erkenntniswert, Erkenntnistat, Art des Gegebenseins und Oratio obliqua bzw. epistemischen Kontexten wie folgt zusammenfassen :

(i) Beide Sätze (i) und (ii) sind voneinander verschieden im Sinne genau dann wenn (i) und (ii) voneinander in der Art des Gegebenseins des Bezeichnetens (d.h. Wahrheitswerts) abweichen : 
Prinzip (I) : $\overline{\text { (i) }} \neq \overline{(i i)} \longleftrightarrow A(\mathrm{i}) \neq A(\mathrm{ii})$.

(ii) Beide Sätze (i) und (ii) sind voneinander verschieden im Erkenntniswert, wenn es z.B. keiner besonderen Erkenntnistat bedarf, um die Wahrheit eines Satzes (i) zu erkennen, während eine besondere Erkenntnistat erforderlich ist, um die Wahrheit eines anderen Satzes (ii) einzusehen (d.h. (i) ist analytisch bzw. erläuternd im Kantischen Sinne, während (ii) synthetisch bzw. erweiternd ist) :

Prinzip (II) : $T^{+}$(ii) $\& T^{-}(\mathrm{i}) \longrightarrow E(\mathrm{i}) \neq E(\mathrm{ii})$.

(iii) Die Verschiedenheit im Erkenntniswert von Sätzen (i) und (ii) ist die notwendige und hinreichende Bedingung (als die ratio essendi und cognoscendi) für die Verschiendenheit des Sinnes von Sätzen, sofern (i) und (ii) keine ,token reflexivéAusdrücke enthalten :

Prinzip (III) $: E($ i $) \neq E($ ii) $\longleftrightarrow \overline{(\mathrm{i})} \neq \overline{(\mathrm{ii})}$.

(iv) Man kann nicht immer sagen, daß die Verschiedenheit des Sinnes die hinreichende Bedingung (als die ratio essendi) für die Verschiedenheit im Erkenntniswert ist :, $\bar{\phi} \neq \bar{\psi} \longrightarrow E(\phi) \neq E(\psi)$ “ ist nicht immer allgemeingültig.

$(v)$ Die Möglichkeit der Verschiedenheit in der Glaubenshaltung ist eine notwendige und hinreichende Bedingung für die Verschiedenheit des Sinnes, sofern , $\phi^{\prime}$ und ,$\psi^{6}$ keine ,token reflexive'-Ausdrücke enthalten :

Prinzip (IV) : $\bar{\phi} \neq \bar{\phi} \longleftrightarrow \diamond \exists x(B x \bar{\phi} \&\urcorner B x \bar{\phi})$.

(vi) Auf Grund der Prinzipien (III), (IV) und den Klausel (iv) zum Prinzip (III), kann man auch sagen, daß die Möglichkeit der Verschiedenheit in der Glaubenshaltung im allgemeinen eine notwendige Bedingung für die Verschiedenheit im Erkenntniswert ist :

Prinzip (V) : $E(\phi) \neq E(\psi) \longrightarrow \diamond \exists x(B x \bar{\phi} \&\urcorner B x \bar{\psi})$.

(vii) Auf Grund der Prinzipien (I) und (IV) kann man auch mindestens sagen, daß die Möglichkeit der Verschiedenheit in der Glaubenshaltung im allgemeinen eine notwendige Bedingung für die Verschiedenheit der Art des Gegebenseins ist :

Prinzip (VI) : $A(\phi) \neq A(\psi) \longrightarrow \diamond \exists x(B x \bar{\phi} \&\urcorner B x \bar{\psi})$.

(viii) Auf Grund der Prinzipien (II) und (V) kann man auch sagen, daß die $-261-$ 
Möglichkeit der Verschiedenheit in der Glaubenshaltung eine notwendige Bedingung für die Notwendigkeit bzw. Nicht-notwendigkeit einer besonderen Erkenntnistat ist, um die Wahrheit der beiden Sätzen zu erkennen :

Prinzip (VII) : $\left.T^{+}(\mathrm{ii}) \& T^{-}(\mathrm{i}) \longrightarrow \diamond \exists x(B x \overline{(\mathrm{i})} \&\urcorner B x \overline{(\mathrm{ii})}\right)$.

Man könnte also auf Grund der Bemerkung (iv) zum Prinzip (III) vermuten, daß beide Sätze,$\phi^{6}$ und,$\psi^{6}$ einander sinngleich sein könnten, wenn , $\phi^{6}$ und,$\psi^{6}$ auch voneinander verschiedene Erkenntniswerte haben, z.B. wenn indexikalische Ausdrücke enthalten sind.

Zweitens kann man auf Grund der Klausel zum Prinzip (IV) sagen, daß die Verschiedenheit des Sinnes nicht das einzige semantische Gegenstück für die Möglichkeit der Verschiedenheit in der Glaubenshaltung ist. Ein anderes semantischen Gegenstück könnte sich auf die Möglichkeit der Verschiedenheit in der Glaubenshaltung beziehen.

Drittens ist die Verschiedenheit der Art des Gegebenseins des Bezeichneten auf Grund der Klausel zum Prinzip (IV) nicht das einzige epistemische Gegenstück für die Möglichkeit der Verschiedenheit in der Glaubenshaltung. Es könnte ein anderes epistemischen Gegenstück geben. Diese drei Punkte würden aber sich eng auf die eigentümliche semantische Rolle der ,token reflexive'-Ausdrücke beziehen. ${ }^{(18)}$

\section{Anmerkungen}

*Dies ist ein Teil des Manuskripts für das Vortrag, gehalten im Colloquium des philosophischen Seminars an der Universität Göttingen, am 23.10.1980.

Besonderen Dank schulde ich Professor G. Patzig, der die erste Fassung dieser Arbeit gelesen hat, und die Arbeit durch mehrmalige Diskussionen und Anregungen gefördert hat. Dank habe ich abzustatten der Alexander von Humboldt-Stiftung, die diese Arbeit von 1979 bis 1980 durch ein Stipendium unterstüzt hat.

(1) Frege sagt aber im Brief an Husserl wie folgt:

„Was ich früher beurteilbaren Inhalt nannte, habe ich nun in Gedanken und Wahrheitswert zerlegt. Das Urteilen im engeren Sinne könnte man kennzeichen als ein Uebergehen vom Gedanken zum Wahrheitswerte." (Brief an E. Husserl am 24.5. 1891 in [WB] 96.)

Andererseits sagt Frege im Brief an $\mathrm{Ph}$. Jourdain wie folgt:

„Ein Satz drückt einen Gedanken aus und bezeichnet dessen Wahrheitswert. Vom Urteile kann man eigentlich weder sagen, daß es bezeichnet, noch daß es ausgedrückt werde. Wir haben darin allerdings einen Gedanken, und dieser kann ausgedrückt werden; Wir haben aber mehr, nämlich die Anerkennung der Wahrheit dises Gedankens : ... Statt „beurteilbarer Inhalt" kann man auch „Gedanke“ sagen." (Brief an Ph. Jourdain in [WB] 119-120.)

(2) Thiel [1965] 136.

(3) Frege sagt wie folgt: 
„Es scheint mir ein objektives Kriterium notwendig zu sein, um einen Gedanken als denselben wiederzuerkennen, weil ohne ein solches eine logische Analyse nicht möglich ist. Um nun zu entscheiden, ob der Satz $A$ denselben Gedanken ausdrücke wie der Satz $B$, scheint mir folgendes Mittel allein möglich zu sein, wobei ich annehme, daß keiner der beiden Sätze einen logisch evidenten Sinnbestandteil enthalte. Wenn nämlich sowohl die Annahme, daß der Inhalt von $A$ falsch und der von $B$ wahr sei, als auch die Annahme, daß der Inhalt von $A$ wahr und der von $B$ falsch sei, auf einen logischen Widerspruch führt, ohne daß man zu dessen Feststellung zu wissen braucht, ob der Inhalt von $A$ oder von $B$ wahr oder falsch sei, und ohne daß man dazu anderer als rein logischer Gesetze bedarf, so kann zum Inhalte von $A$, soweit er fähig ist, als wahr oder falsch beurteilt zu werden, nichts gehören, was nicht auch zum Inhalte von $B$ gehörte; denn für einen solchen Ueberschuss fehlte es an jeder Begründung im Inhalte von $B$, und der Voraussetzung nach wäre ein solcher Ueberschuss auch nicht logisch evident. Ebenso kann bei unserer Annahme zum Inhalte von $B$, soweit er fähig ist, als wahr oder falsch beurteilt zu werden, nichts gehören, was nicht auch zum Inhalte von $A$ gehörte. Was also an den Inhalten von $A$ oder $B$ als wahr oder falsch beurteilbar ist, stimmt überein, und dies kommt für die Logik allein in Betracht, und das nenne ich den von $A$ ebenso wie von $B$ ausgedrückten Gedanken." (Brief an Husserl am 9.12.1906 in [WB] 105-6) Freges hauptsächliche Argument könnte man die natürliche Deduktion Gentzens auf folgender Weise formalisieren durch:

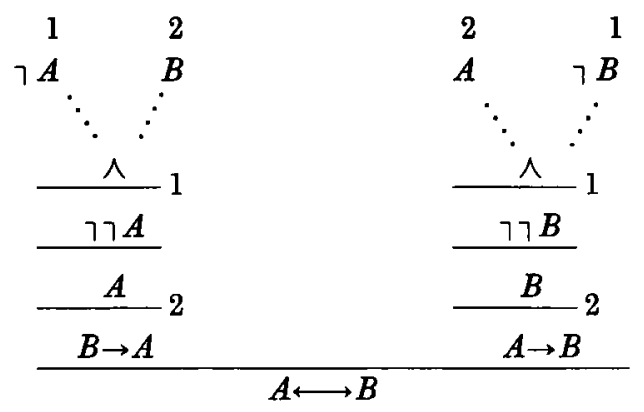

(Hierbei bezeichnet , $\wedge$ ' den logischen Widerspruch.)

(4) In der Tat gibt Frege nach disesem Kriterium einige Beispiele für sinngleichen Sätze in "Gedankengefüge" (39-50) an, worauf Thiel schon hingewiesen hat. (Thiel [1964] 143) Vgl. Bartlett, 20.

(5) Carnap $[\mathrm{MN}] \S 2,10$; Patzig, "Satz und Tatsache“ in : „Argumentationen“, Festschrift für J. König, 1964, 170-191. Neudruck in Patzig [1970] und [1980].

(6) Carnap $[\mathrm{MN}] \S 5,23$.

(7) Z.B. Kripke [1959], [1963].

(8) „Die verschiedenen Gedanken, die sich so aus demselben Satze ergeben, stimmen freilich in ihrem Wahrheitswerte überein, d.h. wenn einer von ihnen wahr ist, sind sie alle wahr, und wenn einer von ihnen falsch ist, sind sie alle falsch. Dennoch ist ihre Verschiedenheit anzuerkennen". (Ged. 66)

(9) Frege bietet dadurch die folgende zweite engere notwendige Bedingung für Gleichheit von Gedanken an, daß er den Sinn von der Bedeutung unterscheidet und daß er auch den Gedanken als den Sinn eines Satzes betrachtet:

„Wenn man nun in einer Zeichenverbindung , $\phi(A)^{4}$, die eine Bedeutung hat, ein

Zeichen,$A^{\prime}$ durch ein anderes,$\Delta^{\prime}$ von derselben Bedeutung ersetzt, so wird die neue Zeichenverbindung,$\phi(\Delta)^{4}$ offenbar dasselbe bedeuten wie die ursprüngliche, $\phi(A)^{\text {c }}$. 
Wenn aber der Sinn von , $\Delta^{\prime}$ vom Sinne von,$A^{\prime}$ abweicht, so wird im allgemeinen auch der Sinn von , $\phi(\Delta)^{\prime}$ vom Sinne von,$\phi(A)^{\prime}$ abweichen." ([Peano] 370)

Weiter wendet er diese auf einige Beispiele an:

,Wenden wir dies auf den Satz, $3+1=2.2^{\star}$ an, indem wir für, $3+1^{6}$ der Reihe nach die gleichbedeutenden Zeichen $, 1+3^{k}, 2+2^{c}, 2.2^{c}$ einsetzen. So erhalten wir die Sätze

$$
\begin{aligned}
& , 1+3=2.2^{\prime} \\
& , 2+2=2.2^{c} \\
& , 2.2=2.2^{\prime}
\end{aligned}
$$

die alle dieselbe Bedeutung haben müssen, und diese nenne ich das Wahre, während sie Verschiedenes ausdrücken. Den Sinn eines Satzes nenne ich einen Gedanken. Diese Sätze drücken also verschiedene Gedanken aus." ([Peano] 370)

(10) Es ist eine bekannte Hauptthese. Freges, daß die gewöhnliche Bedeutung eines Wortes von seiner ungeraden und sein gewöhnlicher Sinn von seinem ungeraden Sinne verschieden ist und ebenso bekannt ist, daß er außerdem die Wendung , der Sinn des

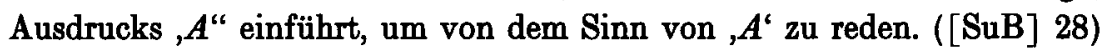

Wenn wir überdies akzeptieren, daß jeder Ausdruck nicht nur seine gewöhnliche Bedeutung, sondern auch seine ungerade Bedeutung (d.h. seinen gewöhnlichen Sinn) als seinen Wert hat wie in Churchs, logic of sense and denotation' (Church [1954], [19734]), dann kann man das sogenannte Prinzip der Ersetzbarkeit von der Bedeutung auf den Sinn übertragen, d.h.,

"Wenn man in einer Zeichenverbindung,$\phi(A)^{4}$, einen die eine [ungerade] Bedeutung [d.h. Gedanken] hat, ein Zeichen , $A^{\prime}$ durch ein anderes,$\Delta^{\prime}$ von derselben [ungeraden] Bedeutung [d.h. Sinn] ersetzt, so wird die neue Zeichenverbindung,$\phi$ $(\Delta)^{\mathfrak{l}}$ offenbar dasselbe [d.h. denselben Gedanken] bedeuten wie die ursprüngliche $, \phi(A)^{c}, "$ (Vgl. [Peano] 369-370)

(11) Carnap [MN] § 14, 56f. Vgl. Churchs Alternative [0], [1], [2] (Church [1954], [1973-74]).

(12) [SuB.] $35 ;$ [Ged.] 65.

(13) Kant [K.r.V.] B28, B33.

(14) Vgl. Frege $[G L A] \S 3$.

(15) Siehe meinen Aufsatz: „Kritische Bemerkungen zur Theorie Freges über ,token reflexive' Ausdrücke', das Vortrag, gehalten im Colloquium des philosophischen Seminars an der Universität Göttingen am 23.10.1980.

(16) Vgl. [Ged. 64] und siehe auch meine Aufsätze: „Kritische Bemerkungen zur Theorie Freges über ,token reflexive" Ausdrücke" und „Frege on Indexicals“

(17) Siehe meinen Aufsatz: „Kritische Bemerkungen zur Theorie Freges über ,token reflexive "Ausdrücke".

(18) Siehe meine Aufsätze: „Kritische Bemerkungen zur Theorie Freges über ,token reflexive“ Ausdrücke“ und „Frege on Indexicals".

\section{Literaturverzeichnis}

Schriften Freges :

Begriffsschrift $[B S]$, Halle, 1879.

Die Grundlagen der Arithmetik [GLA], Breslau, 1884.

Grundgesetze der Arithmetik [GGA], Jena, Bd. I, 1893, Bd. II, 1903. 
„Funktion und Begriff“ [FuB] : Vortrag, gehalten in der Sitzung der Jenaischen Gesellschaft für Medizin und Naturwissenschaft, Jena (1891) 1-31, Neudruck in [FBB] und in [KS].

„Ueber Sinn und Bedeutung" [SuB], in Zeitschrift für Philo. und phi. Kritik 100 (1892), 2550, Neudruck in [FBB] und [KS].

„Ausführungen über Sinn und Bedeutung" (1892-95) [ASB] in [LS] 25-34 und in [NS] 128136.

„Ueber Begriff und Gegenstand“ [BG], in Vierteljahrsschrift für wissenschaftliche Philosophie, XVI (1892), 192-205, Neudruck in [FBB] and in [KS].

"Ueber die Begriffsschrift des Herrn Peano und meine eigene" [Peano] in : Berichte über die Verhandelungen der königlich sächsischen Gesellschaft der Wissenschaften zu Leibzig, Math. und phys. Klasses, XLVIII (1897), 361-378, Neudruck in [KS].

"Logik" (1897) in [LS] 35-73 und in [NS] 137-163.

„Der Gedanke“ [Ged]. in Beitr. zur philos. des deutschen Idealismus 2 (1918-1919) 58-77, Neudruck in [LU] und in [KS].

"Gedankengefüge ${ }^{6 \text { in }}$ in Beitr. zur philos. des deutschen Idealismus 3 (1923-26) 36-51, Neudruck in [LU] und in [KS].

Funktion, Begriff, Bedeutung [FBB] Hrsg. von G. Patzig, Göttingen, 1962, ${ }^{5} 1980$.

Logische Untersuchungen [LU] Hrsg. von G. Patzig, Göttingen, 1966, ${ }^{2} 1976$.

Kleine Schriften [KS] Hrsg. von I. Angelelli, Darmstadt, 1967.

Nachgelassene Schriften [NS] Bd. I Hrsg. von H. Hermes, F. Kambartel und F. Kaulbach, Hamburg, 1969.

Schriften zu Logik und Sprachphilosophie [LS] Hrsg. von G. Gabriel, Hamburg, 1971.

Wissenschaftlicher Briefwechsel [WB] Hrsg. von G. Gabriel, H. Hermes, F. Kambartel, Chr. Thiel u. A. Veraart, Hamburg, 1976.

Sekundärliteratur :

Bartlett, J.M. : Funktion und Gegenstand, Philo. Diss., München, 1961.

Carl, W. : Existenz und Prädikation, München, 1974.

Carnap, R. : [MN] Meaning and Necessity, Chicago, ${ }^{2} 1956$.

Church, A. : : [IML] Introduction to Mathematical Logic, vol. I, 1956.

ders. $\quad:$ : 1951$]$ "A Formulation to the Logic of Sense and Denotation" in Structure, Method and Meaning Hrsg. von S. Langer et al. 1951, 3-24.

ders. $\quad: \quad$ [1954] "Intensional Isomorphism and Identity of Belief", Philo. Studies, 5, n. 5 (1954), 65-73.

ders. $\quad:$ [1973-4] "Outline of a revised Formulation of the Logic of Sense and Denotation", pt. I, Nous 7, n. 1 (1973) 24-33; pt. II Nous 8, n. 2 (1974), 135-156.

Dummett, M. : : 1973] Frege, Philosophy of Language, London, 1973.

ders. $\quad:$ : $\quad 1978]$ Truth and other Enigmas, London, 1978.

Kant, I. $\quad$ : [K.r.V.] Kritik der reinen Vernunft, ${ }^{2} 1787$.

Kripke, S. : [1959] "A Completeness Theorem in Modal Logic", JSL. 24, n. 1 (1959), 114.

ders. $\quad$ : [1963] "Semantical Considerations on Modal Logic", in Acta Philosophica Fennica XVI, 1963, 83-94.

Nomoto, K. : "Frege's Semantics and Ontology", Formal Approaches to Natural Language, Proceedings of the 2nd Colloquium on Montague Grammar and Related Topics, Tokyo, 1982.

ders. $\quad$ : "Frege on Indexicals", The Annals of the Japan Association for Philosophy of Science, vol. 6, No. 5, 1985.

ders. $\quad$ : Frege's Philosophy of Language (in Japanese), 402 pp., Keiso-Syobo, Tokyo, 
1986.

ders. $\quad$ : The Development of Contemporary Logical Semantics-From Frege to Kripke- (in Japanese), 416 pp., Iwanami-Shoten, Tokyo, 1988.

ders. $\quad$ : „Kritische Bemerkungen zur Theorie Freges über ,token reflexive Ausdrück-

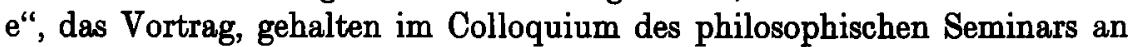
der Universität Göttingen, am 23.10.1980. (erscheinend)

Patzig, G. : : [1966] "Gottlob Frege und die $\gg$ Grundlagen der Arithmetik «" in Neue Deutsche Hefte, 13 (1966) 53-68.

ders. $\quad$ : [1970] Sprache und Logik, Göttingen, 1970.

ders. $\quad:$ : $\quad 1980]$ Tatsachen, Normen, Sätze, Stuttgart, 1980.

ders. : [1981] "Gottlob Frege" in Klassiker der Philosophie, Bd. II, Hrsg. von Höffe, O., München, 1981.

Schirn, M. (Hrrsg.) : Studien zu Frege I-III, Stuttgart-Bad Cannstatt, 1976.

Stegmüller, W.: Hauptströmungen der Gegenwartsphilosophie, Bd. I, ${ }^{6} 1978$, Bd. II, ${ }^{6} 1979$.

Thiel, Ch. : [1965] Sinn und Bedeutung in der Logik Gottlob Freges, Meisenheim am Glan, 1965.

ders. $\quad$ : [1972] "Gottlob Frege" in Gmundprobleme der großen Philosophen, Philosophie der Gegenwart I, 1972, 9-44. 